\title{
Cryo-EM Study of Chaperonin Mm-Cpn's Conformational Heterogeneity under Different ATP Conditions
}

\author{
Yanyan $\mathrm{Zhao}^{1}$ and Wah Chiu ${ }^{1 *}$ \\ 1. Graduate Program of Biophysics, Stanford University Medical School, Stanford, CA, USA. \\ * Corresponding author: wahc@stanford.edu
}

Correct folding of proteins is essential to their functions. Failure to fold into the correct state usually generates inactive proteins, but in some cases misfolded proteins can form toxic aggregates inside the cell. In some instances, toxic aggregates lead to pathological conditions like Alzheimer's disease, Parkinson's disease and Huntington's disease. Chaperonin plays a key role in rescuing misfolded proteins and preventing the formation of potentially toxic aggregates. Chaperonins form a two back-to-back stacked ring structure with central cavities for protein folding. For Group II chaperonin Mm-Cpn, each ring contains eight chemically identical subunits, and each subunit consists of an equatorial, intermediate and apical domain [1]. The subunit can undergo a hinge motion with a pivot point at the interface between the apical and intermediate domains. These three domains reorient upon ATP hydrolysis to close the ring. The ring movements are coordinated in time and space via very complex allosteric regulation. This synchronized ring action depends on communication between individual subunits [2]. A major question in understanding ATP-driven protein folding machines is how the subunits communicate with each other at different steps of ATP hydrolysis (binding, enzymatic cleavage, and products release) resulting in the ring movement. Since it is impractical to synchronize these biochemical steps in an ensemble of Mm-Cpn particles, it yields a variety of molecular species in different biochemical and possibly structure states. Here, we take advantage of cryo-EM technique to study the structure variation of Mm-Cpn in different ATP conditions. Flash freezing allows us to capture the reactants and reaction products in their native states by embedding the sample in vitreous ice state. The extent of structural heterogeneity in the sample is determined by advanced image processing method with single particle images [3].

In this study, we prepared Mm-Cpn structures under three different conditions including different concentrations and incubation period of $\mathrm{ATP}$ and $\mathrm{ATP}-\mathrm{AlF}_{6}$ respectively. We imaged these samples in a $300 \mathrm{keV}$ cryoEM (JEM3200FSC and TF Krios) on a direct electron detector (Gatan K2). Movie frames were aligned with MotionCor2 and CTF was estimated with Gctf. To demonstrate our computational method of analysis, we present here a frame aligned micrograph of Mm-Cpn with ATP along its 2D class averages as exemplified in Fig $1 \mathrm{~A}$.

We used Relion2 and relion3 software to perform particle picking, 2D and 3D classification analysis and reconstruction. We found that $\mathrm{Mm}-\mathrm{Cpn}$ with $\mathrm{ATP}-\mathrm{AlF}_{6}$ data provide the most structurally homogeneous data set resulting in a $3.5 \AA$ resolution map in closed form with a D8 symmetry imposition. On the contrary, the data set of Mm-Cpn with ATP showed large structural heterogeneity. To illustrate our computational method to analyze this heterogeneous data set, we describe here the particle data set with high ATP concentration ( $5 \mathrm{mM})$. Our first step of data classification found $\sim 70 \%$ of our 140,000 particle images in one population with both rings closed similar to the one observed with ATP-AlF 6 . The refined map from this subset of data was determined at $3.4 \AA$ with D8 symmetry. Most of the side chain and the nucleotide densities are visible in this map. Figure 1B shows the ATP and $\gamma$ phosphate in the binding pocket. These observations suggest that the structure of this subset of particles should represent the MmCpn in a true ATP binding state. 
When we carried out a further analysis of the remaining $30 \%$ of the particle images, we computationally divided the two rings in each particle image and subject the rings image intensity to further classification. With another step of 3D focused classification into 5 classes, we found that there are different extents of ring openings from widely to partially opened and closed configurations in the dataset of $\mathrm{Mm}-\mathrm{Cpn}$ as exemplified in Figure $1 \mathrm{C}$ with two of these classes at $3.8 \AA$ resolution. These data demonstrate different structure snapshots of Mm-Cpn undergoing a dynamic motion of a lid closure or opening in a ATP hydrolysis process. Our structure observations show the usefulness of cryoEM to dissect structure heterogeneity in a single biochemical specimen and has the potential to deduce more detailed mechanism of multiple steps and structures in a biochemical process carried out by a molecular machine [4].

\section{References:}

[1] CR Booth et al., Nat Struct Mol Biol 15(7) (2008), p. 746.

[2] J Zhang et al., Nature 463(7279) (2010), p. 379.

[3] SH Scheres, Methods Enzymol 579 (2016), p. 125.

[4] The authors acknowledge support from NIH grants P41GM103832 and S10OD021600.

\section{A}
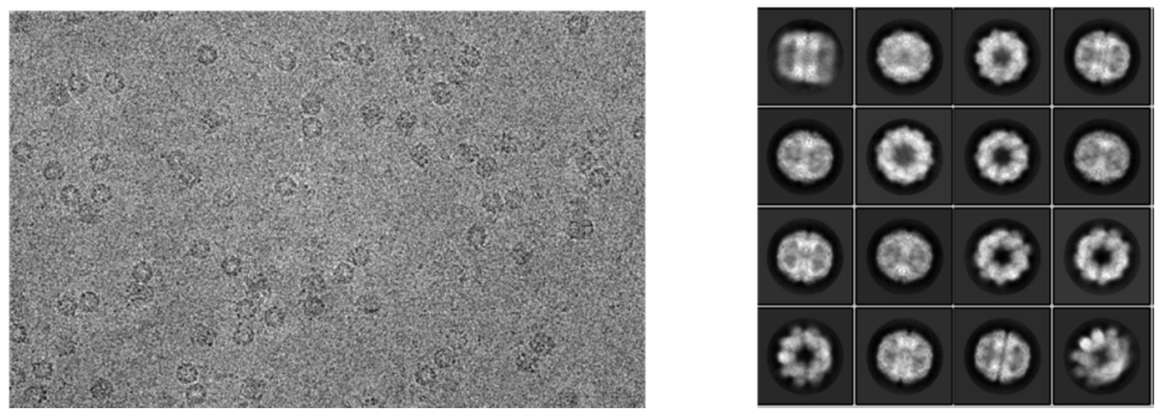

B

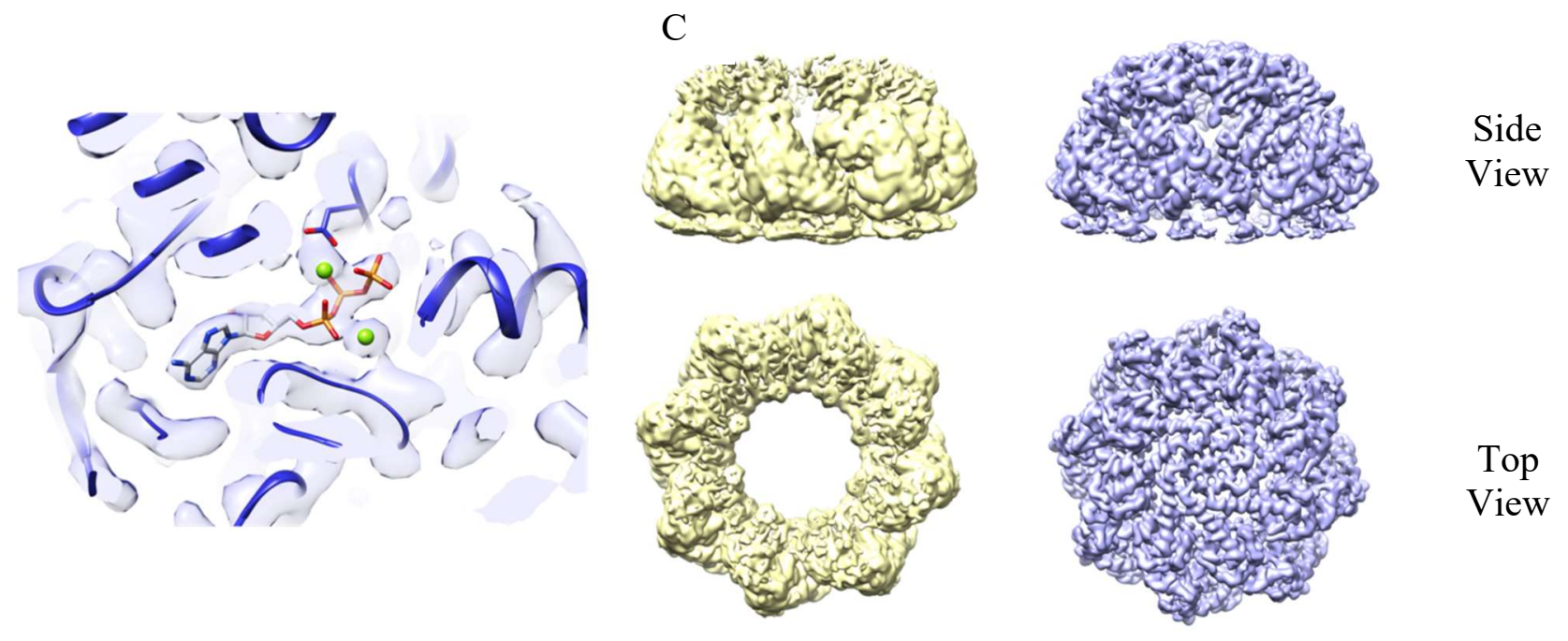

Figure 1. (A) A representative drift-corrected micrograph of the Mm-Cpn under low ATP condition taken

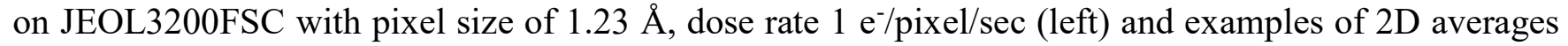
(right). (B) Nucleotide density with the model of ATP fit in the binding pocket of the closed form of MmCpn under high ATP condition. (C) Mm-Cpn under high ATP condition has structural heterogeneity in the ring opening. Partially open rings (yellow) are reconstructed with 31,000 particles of single ring while closed rings (blue) from 26,000 particles of single ring. 